livraisons

d'Histoire

de l'Architecture

\section{Livraisons de l'histoire de l'architecture}

41 | 2021

L'architecte en son agence

\title{
L'apprentissage de l'architecture en « agence » : la formation de Jean-Baptiste Dufart (1750-1820) à Bordeaux, 1768-1780
}

The learning of architecture in "agency": the formation of Jean-Baptiste Dufart (1750-1820) in Bordeaux, 1768-1780

Das Erlernen von Architektur in der "Agence": zur Ausbildung des Jean-Baptiste Dufart (1750-1820) in Bordeaux (1768-1780)

Laurence Chevallier

\section{OpenEdition}

Journals

Édition électronique

URL : https://journals.openedition.org//ha/3110

DOI : 10.4000/lha.3110

ISSN : 1960-5994

Éditeur

Association Livraisons d'histoire de l'architecture - LHA

Référence électronique

Laurence Chevallier, «L'apprentissage de l'architecture en « agence » : la formation de Jean-Baptiste

Dufart (1750-1820) à Bordeaux, 1768-1780 », Livraisons de l'histoire de l'architecture [En ligne], 41 |

2021, mis en ligne le 15 juin 2021, consulté le 18 juin 2021. URL : http://journals.openedition.org//ha/

3110 ; DOl : https://doi.org/10.4000//ha.3110

Ce document a été généré automatiquement le 18 juin 2021.

Tous droits réservés à l'Association LHA 


\title{
L'apprentissage de l'architecture en « agence »: la formation de Jean- Baptiste Dufart (1750-1820) à Bordeaux, 1768-1780
}

\author{
The learning of architecture in "agency": the formation of Jean-Baptiste Dufart \\ (1750-1820) in Bordeaux, 1768-1780 \\ Das Erlernen von Architektur in der "Agence": zur Ausbildung des Jean-Baptiste \\ Dufart (1750-1820) in Bordeaux (1768-1780)
}

\section{Laurence Chevallier}

Ces dernières années, plusieurs publications ont fait progresser nos connaissances sur le quotidien et le fonctionnement du travail des architectes ${ }^{1}$. Dans ce contexte, il nous a semblé pertinent d'interroger "l'agence " comme un lieu privilégié, au XVIII ${ }^{\mathrm{e}}$ siècle, pour la formation de l'architecte. Pour ce faire, nous suivrons la formation d'un architecte, celle de Jean-Baptiste Dufart (1750-1820) à Bordeaux, entre 1768 et 1780 . En effet, dans les agences de Richard-François Bonfin (1731-1814) et de Victor Louis (1731-1800), Dufart parachève sa formation de dessinateur et apprend l'architecture. L'historiographie locale a retenu que les architectes bordelais de l'époque moderne s'étaient essentiellement formés par voie corporatiste ou dans des écoles académiques ${ }^{2}$. Cette littérature spécialisée a ainsi appréhendé les lieux du travail collaboratif ${ }^{3}$, mais assez peu l'agence de l'architecte et encore moins l'agence envisagée comme un lieu d'apprentissage et cela alors même que cette formation "sur le tas " était alors très répandue ${ }^{4}$. Ceci s'explique probablement parce que les apprentissages dans les écoles académiques et par voie de corporatisme ont produit des témoignages et des documents aujourd'hui exploitables, ce qui n'a pas été systématiquement le cas avec la formation en agence. Or, ces documents archivistiques existent dans le cas de Dufart et - bien qu'incomplets et lacunaires - ils sont éclairants sur l'apprentissage qu'il a reçu dans les deux agences où il travaille, entre 1768 et $1780^{5}$. Partant de ce matériau, peuton alors mieux comprendre comment cette formation était possible alors que 
l'architecte était l'employeur et, l'aspirant architecte, un employé salarié ? Peut-on également mieux saisir les échanges et les relations engagés entre le chef du bureau et son subalterne? Quelles formes pouvaient prendre les « leçons »? Quelle place tenait le dessin dans ce type de formation? Quelles opportunités offrait cet apprentissage ? Ce sont ces ébauches de questions que cet article se propose d'appréhender.

\section{Une formation « sur le tas »}

2 Né à Bayonne, Jean-Baptiste Dufart reçoit un premier enseignement de dessin, et plus spécifiquement de tracé et de géométrie, auprès de son père, Jean Dufart (inc.), charpentier de navire ${ }^{6}$. Il quitte le port bayonnais et arrive à Bordeaux dans le courant de l'année 1768. À cette époque, la capitale girondine connaît une importante prospérité économique. La ville est atteinte par une «fièvre $»^{7}$ de construction et un jeune talent pouvait espérer y trouver du travail et de nombreuses opportunités. C'est ainsi que dès son arrivée, Dufart intègre d'abord le bureau municipal de l'ingénieurarchitecte en chef de la Ville, Richard-François Bonfin ${ }^{8}$. En 1773, il entre dans le bureau de l'architecte parisien Victor Louis (1731-1800) ouvert à Bordeaux pour la construction du nouveau théâtre de la ville : le Grand-Théâtre (1773-1780)9.

Dans ces deux bureaux, Jean-Baptiste Dufart est un employé, salarié, et ces deux architectes ne sont pas ses professeurs mais ses patrons. Dans le bureau de la Ville, le jeune dessinateur perçoit un mandement annuel de 600 livres $^{10}$. Chez Victor Louis, ses émoluments passent à 800 livres par $a^{11}$.

\section{La pratique quotidienne du dessin}

$4 \quad$ Au sein de ces deux agences, l'activité principale de Dufart est le recopiage au propre des dess(e)ins des deux architectes. On peut aisément supposer que si, au tout début, il est guidé et travaille à recopier des dessins simples, rapidement il poursuit son apprentissage par des dessins plus complexes : esquisses, dessins préparatoires, épures, dessins techniques ou encore dessins d'exécution. Pour faire ce travail de mise au propre, Dufart doit apprendre et assimiler les logiques de compositions spatiales et structurelles ainsi que les usages de l'édifice, c'est-à-dire l'intégration, les proportions, la distribution, les circulations, la volumétrie, etc. Cette pratique quotidienne permet la compréhension et l'analyse du projet de construction car il ne s'agit pas seulement d'une simple et fidèle mise au propre mais bien du fondement principal de la formation à l'architecture, reçu par Dufart dans ces deux agences.

5 À côté de cette tâche quotidienne et fondamentale, Dufart a également l'occasion de participer à d'autres activités pour lesquelles la pratique du dessin est tout aussi formatrice. C'est notamment le cas avec l'actualisation du plan de la Sauvetat de la collégiale de Saint-Seurin de Bordeaux, une mise à jour sur laquelle le jeune dessinateur travaille entre 1770 et $1774^{12}$. Pour mener à bien cette mission, que lui a confiée Bonfin, Dufart doit d'abord apprendre et comprendre les "polices", à savoir les règles de justice qui organisent et régissent la vie en société sous l'Ancien Régime. Pour le jeune dessinateur, c'est donc l'occasion d'apprendre à jongler avec les textes de lois, les traditions, les règlements, les décrets... Tout le lourd aspect administratif de la construction sous l'Ancien Régime. Ce plan réactualisé de la Sauvetat doit aussi permettre de réfléchir à la translation du cimetière construit le long de la collégiale. En 
effet, l'église Saint-Seurin est historiquement implantée au cœur d'une nécropole antique d'origine paléochrétienne, qui abrite le plus ancien cimetière de la ville ${ }^{13}$. C'est ici l'occasion pour Dufart de s'imprégner des théories de l'hygiénisme naissant ${ }^{14}$ qui, depuis quelques années, insistent sur l'importance de la propreté publique et personnelle. Les cimetières sont alors considérés comme des lieux sans salubrité publique, aux odeurs et aux abords souvent délicats ; et c'est justement le plus souvent de cela dont se plaignent les voisins de Saint-Seurin.

Quel que soit le type de tâche affectée à Dufart, on comprend bien comment l'apprentissage du métier d'architecte en agence se fait par une pratique quotidienne du dessin et comme l'affirme Daniel Rabreau : « on peut dire sans exagérer que tout l'enseignement de l'architecture au XVIII ${ }^{e}$ siècle repose sur la pratique du dessin ${ }^{15} »$. Et si l'on ne connaît presque rien des dessins exécutés par Dufart pour les deux architectes, le professeur Christian Taillard - spécialiste de Victor Louis - est convaincu qu'une «[...] bonne partie des dessins réalisés pour l'exécution du Grand-Théâtre est probablement de sa main $[. . .]^{16}$.»

\section{La pratique quotidienne du chantier}

7 Dans cette formation faite "sur le tas ${ }^{17}$ ", Dufart est également amené à passer beaucoup de temps sur les chantiers de construction et notamment sur celui du GrandThéâtre, qu'il fréquente quotidiennement entre 1773 et 1780.

Là, le dessinateur peut en profiter pour étudier les questions relatives au choix des matériaux, au processus de construction, à la direction et à l'économie du chantier. Ainsi, Dufart peut-il, par exemple, observer et étudier la réalisation des voûtes plates $d u$ vestibule d'entrée du Grand-Théâtre. L'art du trait et la stéréotomie sont évidemment des affaires sérieuses pour Victor Louis qui, avec ces voûtes déprimées savantes, impressionne ses contemporains. Pour la réalisation de ces voûtes, Dufart a dû aussi être captivé par les compétences et l'habileté des appareilleurs et des tailleurs de pierre qui pouvaient, seuls, rendre cela possible. Cette union entre arts mécaniques et arts libéraux est certainement l'une des importantes leçons retenues par Dufart sur ce chantier.

9 Dufart peut aussi appréhender tout l'univers professionnel de l'art de bâtir et mieux saisir l'implication des différents métiers et acteurs du chantier dans la construction et notamment l'organisation des échanges entre l'architecte et ses collaborateurs. Victor Louis porte, également, une attention scrupuleuse au choix des différentes qualités de pierre nécessaires à la construction de son théâtre ainsi qu'à une gestion rationnelle du chantier, des équipes et de l'achat des différents matériaux ${ }^{18}$. En 1775, Dufart est nommé « [...] inspecteur de la nouvelle salle de spectacle [... ${ }^{19} »$. Cette nouvelle fonction lui permet d'approfondir un peu plus sa formation pratique car en tant qu'inspecteur il doit maintenant surveiller les travaux et la conformité de ceux-ci avec ses propres dessins. Il doit également participer à établir les devis et vérifier la qualité des matériaux livrés.

10 Les archives ne nous permettent pas de connaître, avec plus de précision, les tâches contrôlées par Dufart. Mais, à n'en pas douter, ce chantier est un lieu d'observation unique qui offre à Dufart des leçons d'architecture concrètes et des opportunités d'apprentissages journalières. Il y apprend notamment que le chantier peut être le lieu de la coopération entre les divers corps de métier et surtout entre le concepteur et les 
techniciens qui utilisent alors le dessin, comme un outil, un support, à leurs échanges. Le chantier du Grand-Théâtre est également une occasion privilégiée pour se faire connaître du microcosme de la construction bordelaise avec lequel Dufart travaille et négocie quotidiennement. Enfin, ce prestigieux chantier - ainsi que la réputation de Victor Louis - procure à l'architecte débutant ses premières opportunités professionnelles.

\section{Le « retour à l'antique » triomphant}

Le travail de Jean-Baptiste Dufart dans les agences d'architecture de Bonfin et de Louis l'amène à travailler sur les rares vestiges antiques de la Ville de Bordeaux; une découverte qui est sa seule réelle confrontation avec l'Antiquité. En effet, Dufart n'aura toute sa vie qu'une connaissance livresque de celle-ci car, par faute de moyens, de temps ou d'opportunité, il n'ira jamais à Rome.

\section{Dom Deviennes, I'Histoire de Bordeaux, 1771-1772}

Richard-François Bonfin est le fils de François Bonfin (vers 1690-1750), l'un des collaborateurs de l'équipe versaillaise des Gabriel père et fils, venue à Bordeaux pour la construction de la place Royale (1729-1750). Après le départ de AngeJacques Gabriel (1698-1782), ses adjoints ont la charge de diriger la fin des travaux. À vingt ans, Richard-François succède à son père à la tête de ce chantier, et, en 1751, satisfaits de son travail, les Jurats le nomment, à la suite de la mort de son père, ingénieur de la Ville. À cette place, Bonfin poursuit la diffusion de l'héritage classique des Gabriel, un héritage alors teinté de Rocaille. C'est donc à ce style hybridé que Dufart est originellement confronté. Sa participation à l'entreprise de Dom Devienne, L'histoire de Bordeaux (1752-1772) ${ }^{20}$, est une occasion unique pour lui de se familiariser avec les antiques de la ville et de s'interroger sur le mouvement de « retour à l'antique » qui s'impose alors dans tout le pays. Une lettre, datée du 26 juin 1771, écrite de la main de Dom Devienne, destinée aux Jurats et les informant de l'avancement du travail, ne laisse aucun doute sur la précoce participation de Bonfin au projet: «[...] les dessins des planches qui doivent être gravés sont faits [...]. Monsieur Bonfin a déjà fourni le plan géométral de l'ancien Bordeaux et va livrer incessamment celui du palais Gallien qui représentera l'intérieur et l'extérieur de cet amphithéâtre $[. . .]^{21} »$. Dans une nouvelle missive, toujours destinée aux Jurats, datée du 5 janvier 1772, il est maintenant fait mention de l'intervention de «[...] Monsieur Bonfin et du petit Dufart [...] » pour la réalisation des illustrations ${ }^{22}$. Dufart est alors chargé de réaliser le dessin du plan de l'amphithéâtre antique de Bordeaux, le Palais Gallien, une tâche originellement confiée à Bonfin. Gravée par Pierre-Claude de La Gardette (1743-1782), graveur de Paris $^{23}$, cette planche doit servir d'illustration à l'ouvrage et tout particulièrement au premier chapitre, intitulé Les Préliminaires, et largement consacré au Bordeaux antique, Burdigala, et aux monuments qui le composaient. Bonfin profite de cette entreprise pour confronter Dufart à l'unique vestige antique encore debout dans la ville. (Ill. 1) 
III. 1 : Jean-Baptiste Dufart (dessinateur) et Pierre-Claude de La Gardette (graveur), Plan du Palais Gallien

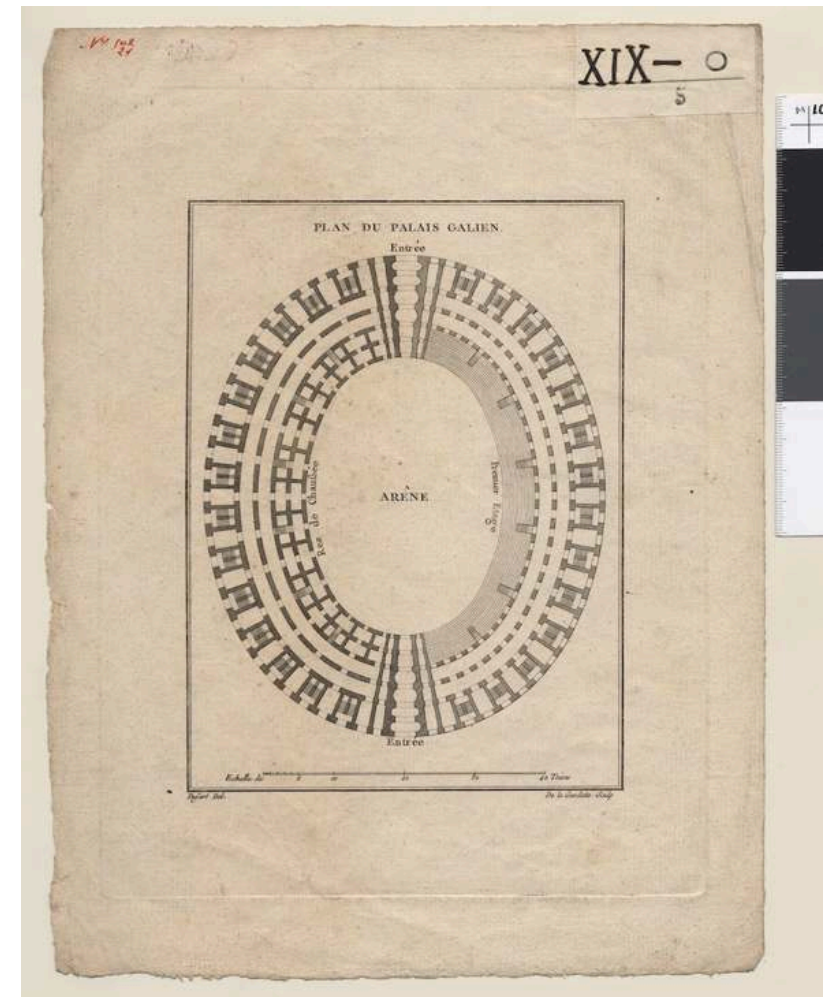

Papier, Archives Bordeaux Métropole, cote XIX/O-5, sans date.

(C) Archives Bordeaux Métropole, Bordeaux Fi XIX 05

La tâche qui attend Dufart est ardue. En effet, il doit lever le plan d'un édifice qui se trouve alors dans un état de ruine très avancée et qui, à l'origine, devait dépasser les 130 mètres dans sa plus grande longueur. Dufart exécute un travail honnête sur l'édifice existant qui permet de rendre l'état de ce bâtiment, à son époque. Pour ce faire, il relève toutes les côtes encore disponibles et restitue ainsi, par calque, plus de la moitié de l'édifice manquant. Cette expérience est primordiale pour Dufart notamment dans le perfectionnement de ses techniques de relevé et de tracé, mais cette entreprise agit également sur lui comme une découverte de l'Antiquité alors qu'il se retrouve à relever, mesurer et dessiner cet édifice antique.

La réalisation de ce dessin nous révèle également comment les contemporains de Dufart entretenaient un rapport contradictoire avec les antiques. En effet, très peu d'années après la publication de ce dessin, de nombreuses personnes sont prêtes à détruire le Palais Gallien pour faire place à une vaste opération de spéculation immobilière et financière. Richard-François Bonfin, lui-même, associé à son collègue François Lhôte (1743-1808), veut faire démolir le vieil amphithéâtre afin de lotir l'immense emplacement laissé disponible par sa destruction. Déjà, du temps de la réalisation du dessin de Dufart, les autorités municipales désiraient faire de ces ruines un espace plus attrayant, peut-être en y installant une place ou un jardin ${ }^{24}$. En réalisant ce dessin, Dufart se retrouve donc confronté à l'une des importantes et nécessaires réalités du monde artistique : son aspect économique. 


\section{Quel retour à l'antique?}

Aux côtés de Victor Louis, Dufart s'imprègne plus fortement encore de ce « retour à l'antique » triomphant. Ainsi, inspiré en grande partie par son propre séjour romain, Louis devient le promoteur à Bordeaux, de ce tout nouveau répertoire de formes et notamment d'une version dite "à la romaine " qu'il développe dans un style décoratif et sophistiqué. Dès lors, Dufart conserve, pour cette époque et son architecture monumentale, une admiration déterminante dans le devenir et l'évolution de ses propres choix esthétiques et stylistiques ${ }^{25}$.

En effet, la carrière d'architecte de Dufart peut se lire comme un catalogue des différents changements du goût entre la fin ${\mathrm{du} X V I I I^{\mathrm{e}}}$ et le début du XIX ${ }^{\mathrm{e}}$ siècle, à Bordeaux. Dans le sillage de Bonfin, ses premières réalisations restent fidèles à ce style hybridé, entre tradition versaillaise et art rocaille. Puis, alors que la ville de Bordeaux, sous l'influence de Victor Louis, connaît le développement d'un retour à l'antique où le modèle romain domine, Dufart choisit, quant à lui, de s'orienter vers davantage de dépouillement en abandonnant progressivement tout décor superflu. D'ailleurs, la grande simplicité du dessin de ses dernières grandes réalisations confirme que cette question le préoccupait particulièrement et Dufart semble prendre définitivement le parti d'une écriture à l'antique sans concession. Dès lors, il marque ses œuvres d'une plus grande sévérité: il accentue l'emploi de plans équilibrés, symétriques et harmonieux où seuls comptent la monumentalité et les jeux sur les volumes architecturaux. Cette écriture favorise l'organisation des masses se suffisant à ellesmêmes et, de fait, annihile alors tout décor devenu superflu ${ }^{26}$ (Ill. 2).

\section{2 : Jean-Baptiste Dufart, façade principale de la Maison Fenwick}

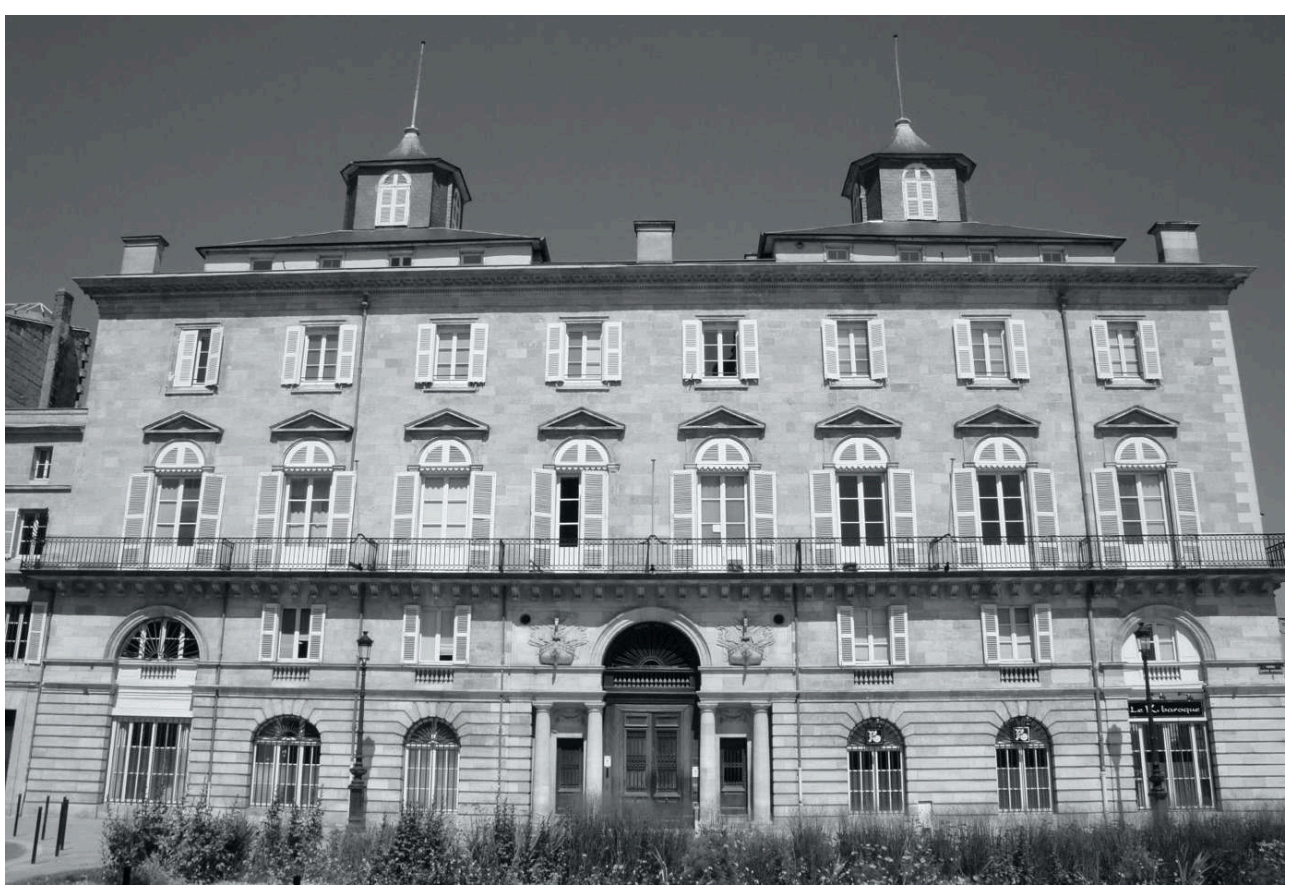

Bordeaux, 1795-1799

(C) Cl. Laurence Chevallier 
17 Pendant les douze années que Dufart passe dans les agences de Richard-François Bonfin et de Victor Louis, il expérimente le travail en agence et le travail sur le chantier, soit une formation à l'architecture qui se fait par la pratique, par le terrain. Cette dialectique dessin/chantier ${ }^{27}$, conception/réalisation est le fondement de l'apprentissage au métier d'architecte que Dufart reçoit dans ces deux agences.

Richard-François Bonfin a joué un rôle déterminant dans les premières années que Dufart passe à Bordeaux. Ainsi, en l'embauchant au sein de son équipe municipale, lui offre-t-il la possibilité d'approfondir et d'améliorer tout son savoir de dessinateur. Chaque intervention de Bonfin devient l'occasion pour Dufart d'appréhender toutes les interactions possibles d'un architecte dans la ville : au niveau privé, pour résoudre un souci d'alignement de façade, au niveau édilitaire, par la construction, la reconstruction ou l'aménagement d'un site, ou bien encore au niveau religieux, pour résoudre, entre autres, des problèmes de police et de possession. Bonfin lègue aussi à Dufart ce goût pour la tradition versaillaise, teintée de rocaille, tout en lui ouvrant les yeux sur la nouvelle mode de « retour à l'antique ». La collaboration avec Victor Louis, rendue possible par l'intervention de Bonfin, est une nouvelle opportunité professionnelle pour Dufart. Il côtoie au quotidien un véritable microcosme artistique d'une grande qualité. Ainsi, peut-il suivre les ouvrages des collaborateurs parisiens de Louis comme ceux du sculpteur Pierre François Berruer (1733-1797) ou encore ceux du peintre Jean-Baptiste Claude Robin (1734-1818). Il peut également, parmi ses collègues de l'agence, se confronter à la prochaine génération d'architectes bordelais et notamment à Gabriel-Joseph Durand (1750-1814) ou à Alexis-Honoré Roché (1757-1828). La renommée de Louis et de son théâtre ne cessent de croître auprès de ses contemporains et c'est sans nul doute grâce à son implication dans cette construction que Dufart obtient l'une des commandes les plus importantes de sa carrière : la réalisation du Théâtre Français (Ill. 3) ${ }^{28}$. 
III. 3 : Jean-Baptiste Dufart, façade principale du Théâtre Français

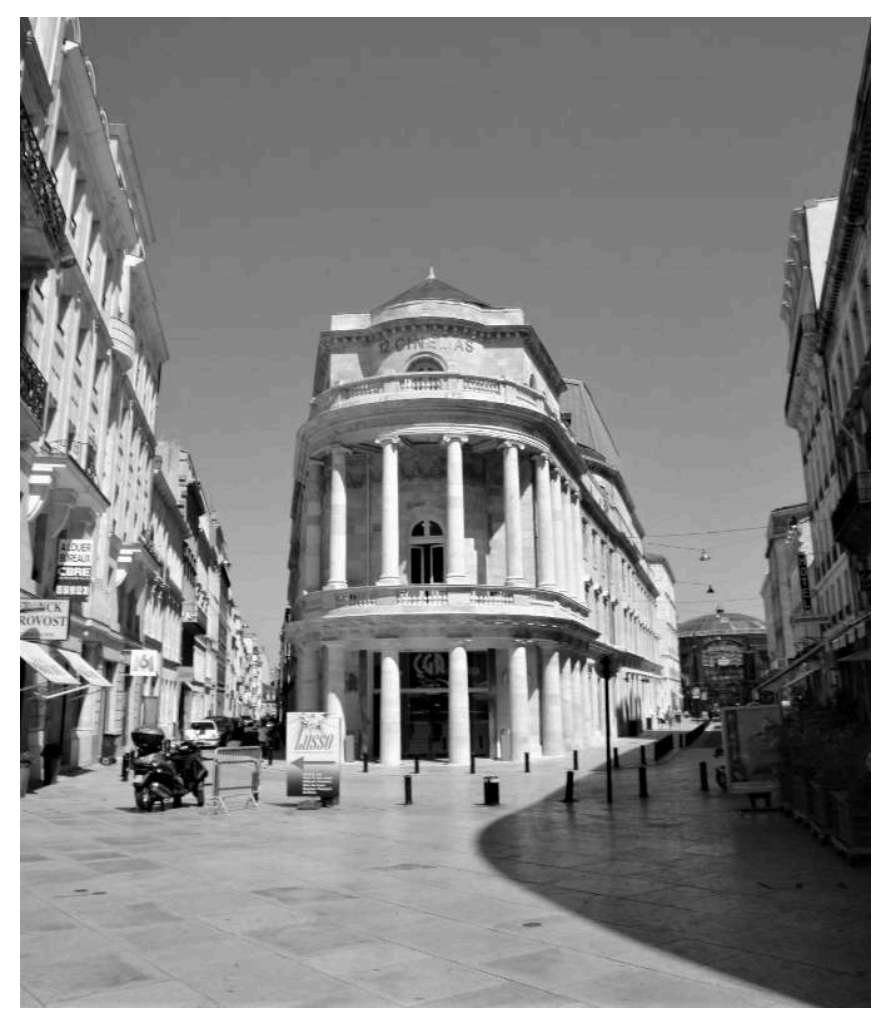

Bordeaux, 1793-1800.

(C) Cl. Laurence Chevallier

19 L'impact et l'apport de Victor Louis sur les débuts de la carrière de Dufart sont indéniables. Si bien, que certains ont été tentés, légitimement, de faire de Dufart l'élève de Louis. S'il est vrai qu'avec ce dernier, Dufart observe et apprend les moindres aspects de la grande architecture, approfondit sa découverte de la nouvelle inspiration antique ainsi que ce goût très particulier pour la direction des chantiers, leurs relations ne sont pas pour autant celles d'un maître avec son élève. Et, même s'il n'est jamais clairement mentionné, le véritable maître de Dufart est Richard-François Bonfin. Ainsi, dès qu'il arrive à Bordeaux, Dufart loge même chez Bonfin. Un document d'archives, concernant les travaux de l'hôtel de ville de Bordeaux, daté du $1^{\mathrm{er}}$ septembre 1770 , conserve le souvenir de cet arrangement: «[...] le sieur Dufart à titre de dessinateur sera également payé une somme de 600 livres pour chacune année [...] lequel le dit sieur Bonfin sera obligé de garder avec lui dans sa maison [... $]^{29} »$. La protection de Bonfin est permanente, il ouvre toutes les portes au jeune dessinateur, y compris celles de l'Académie ${ }^{30}$. Leurs rapports de maître et élève se renforcent avec l'entrée de Dufart dans cette prestigieuse institution. Mais leur lien n'est jamais ouvertement déclaré. Le peintre Pierre Lacour (1745-1814) résume fort bien cette situation quand il prononce, le 11 août 1787, le discours de réception de Dufart à l'Académie : il déclare que Dufart est le « [...] digne élève d'un académicien que nous honorons tous, c'est à lui d'achever son ouvrage, il vous a conduit dans le sanctuaire des arts, qu'il vous guide encore un instant, dans cette nouvelle carrière $[. . .]^{31}$ ».

Finalement, Victor Louis et Richard-François Bonfin ont conjointement joué un rôle majeur dans le long apprentissage de Dufart. Ces deux imposantes personnalités ont également ouvert des portes différentes mais complémentaires à la future carrière 
d'architecte de Dufart. Ainsi, l'un l'a-t-il durablement rapproché de la municipalité alors que l'autre a su lui donner l'envergure nécessaire pour débuter une brillante carrière dans le domaine de l'architecture privée ${ }^{32}$. À la suite des douze années passées dans ces deux agences, Dufart est agréé, en 1787, de l'Académie de peinture, sculpture et architecture civile et navale de Bordeaux. Après des débuts remarqués dans la construction privée, il rejoint les équipes municipales en 1806, où il est nommé ingénieur de la voirie. C'est grâce à cette position officielle que la ville lui confie la réalisation du plus important projet urbanistique de Bordeaux au XIX siècle : le tracé définitif de la distribution et délimitation des terrains du château Trompette.

\section{NOTES}

1. Ces études ont fait des lieux de la pratique architecturale, au même titre que l'atelier de l'artiste, un objet d'étude à part entière en s'intéressant à l'emplacement des différents lieux de travail, à leur organisation spatiale et sociale, aux mobiliers, aux outils et aux instruments qui s'y trouvaient. On peut citer : Alexandre Cojannot, « Du maître d'œuvre isolé à l'agence : l'architecte et ses collaborateurs en France au XVII ${ }^{\mathrm{e}}$ siècle", Perspective, 1/2014, p.121-128; Maxime Decommer, Les Architectes au travail. L'institutionnalisation d'une profession, 1795-1940, Rennes, Presses universitaires de Rennes, 2017, 402 pages; Alexandre Cojannot et Alexandre Gady, Dessiner pour bâtir - Le métier de l'architecte au XVII ${ }^{\mathrm{e}}$ siècle, Paris, Le Passage/Archives Nationales, 2017, 350 pages; Valérie Nègre (dir.), L'art du chantier: construire et démolir $d u X_{V I}^{e}$ au XXI siècle, Paris/Gand, Cité de l'Architecture/Snoeck, 2018, 284 pages ; Les Cahiers de la recherche architecturale urbaine et paysagère, L'agence d'architecture (XVIII ${ }^{e}-\mathrm{XXI}^{e}$ siècle), $\mathrm{n}^{\circ} 9$, dossier thématique coordonné par Gauthier Bolle, Maxime Decommer et Valérie Nègre, décembre 2020, en ligne, https://journals.openedition.org/craup/5318

2. Sur l'apprentissage par voie corporatiste à Bordeaux, on peut lire l'ouvrage de Bernard Gallinato, Les corporations à Bordeaux à la fin de l'Ancien Régime : vie et mort d'un mode d'organisation $d u$ travail, Bordeaux, Presse Universitaires de Bordeaux, 1992, 382 pages et celui de Philippe Maffre, Construire Bordeaux au XVIII siècle. Les frères Laclotte, architectes en société, 1756-1793, Bordeaux, Société archéologique de Bordeaux, collection Mémoires, volume 7, 2013, p. 55. Le corporatisme bordelais est établi dans la ville depuis le XVI ${ }^{\mathrm{e}}$ siècle au moins. Tout au long du $\mathrm{XVIII}^{\mathrm{e}}$ siècle, il s'essouffle jusqu'à perdre le soutien des autorités locales. Pour aller plus loin sur l'enseignement délivré dans l'école académique de Bordeaux, on peut consulter: Laurence Chevallier, "L'Académie de peinture, sculpture, architecture civile et navale de Bordeaux (1768-1793), aux origines de l'enseignement de l'architecture en petite Guyenne », Transversale 2 Histoire : Architecture, paysage, urbain, 2017, p. 9-16.

3. Christian Taillard, Victor Louis (1731-1800): Le triomphe du goût français à l'époque néo-classique, Paris, Presses de l'Université Paris-Sorbonne, 2009, 495 pages; Philippe Maffre, Construire Bordeaux au XVIII' siècle. Les frères Laclotte. Architectes en sociétés (1756-1793), Bordeaux, Société archéologique de Bordeaux, 2013, 443 pages ; Renée Leulier, L'architecte André Portier (1702-1770) et l'art Rocaille à Bordeaux, thèse de doctorat, soutenue sous la direction du professeur Christian Taillard, Université Bordeaux-Montaigne, 2018. 
4. Exception faite, peut-être, des pages consacrées par Christian Taillard au bureau de Victor Louis dans son ouvrage: Victor Louis (1731-1800): Le triomphe du goût français à l'époque néoclassique, Paris, Presses de l'Université Paris-Sorbonne, 2009 p. 178-182 et 414.

5. On dispose notamment de documents intéressants dans les archives de la ville et dans celles de la construction du Grand-Théâtre conservées aux Archives Bordeaux Métropole, et spécifiquement dans les séries AA (Actes constitutifs et politiques de la commune), BB (Administration communale, délibérations de la Jurade, 1402-1790), CC (Finances et contributions) et DD (Biens communaux, Eaux et forêts, Travaux publics, Voirie) et, aux Archives Départementales de la Gironde, dans les séries C (Intendance de Bordeaux) et $\mathrm{G}$ (Clergé séculier).

6. Jean-Baptiste Dufart fait allusion à cette formation paternelle, le samedi 11 août 1787, dans un discours qu'il prononce devant ses amis de l'Académie de peinture, de sculpture et d'architecture civile et navale de Bordeaux : «[...] livré dès mon plus jeune âge à l'étude d'un art important qui réunit l'agréable à l'utile, qui s'attache à procurer le plaisir de la vue et qui ne parvient à son but que par la combinaison des grandeurs et par le mélange des formes [...]», Bibliothèque municipale de Bordeaux (BMB), Ms 1540, registre de l'Académie de Peinture, folio 425, 11 août 1787.

7. François-Georges Pariset et Charles Higounet, (sous la direction de), L'Histoire de Bordeaux, Bordeaux au XVIII siècle, Bordeaux, 1968, p. 702.

8. Les activités de ce bureau sont nombreuses et très diversifiées car l'ingénieur-architecte en chef de la Ville s'occupe « [...] du pavement des voies, de l'alignement des rues du centre-ville, du port et de la distribution de l'eau [...] ». À ce titre, le bureau se charge de «[...] l'entretien et la réparation de tous les monuments publics ; portes de ville, quais, aqueducs, maisons, agence de la ville, verbaux à dresser, états estimatifs et devis des ouvrages neufs et des réparations, le règlement des mémoires des ouvriers et fournisseurs, l'inspection des pavés, des formes et des vents, redressements des rues et nivellements ; plans à lever, rapports à donner [...]» : Archives de Bordeaux Métropole (ABM), série BB, liasse 161, emplois de la ville, s. d.

9. Christian Taillard, Victor Louis (1731-1800): Le triomphe du goût français à l'époque néo-classique, Paris, Presses de l'Université Paris-Sorbonne, 2009, p. 178. L'approbation des plans de Victor Louis, le 18 mai 1773, implique - avant-même - l'ouverture du chantier la mise en place d'un bureau.

10. $A B M$, série $B B$, liasse 162 , emplois de la ville, 1768-1773.

11. $A B M$, série $D D$, liasse $36 \mathrm{~g}$, honoraires du Grand-Théâtre, année 1775, mandement du 10 juin 1775.

12. ADG, série $G$, liasse 1492 , compte de recette et de dépense pour les années 1774-1775, chapitre de Saint-Seurin, folio 16, 20 septembre 1774. ABM, fonds Braquehaye, Ms 315, DUB-DUR : pour la mise à jour de ce plan, Dufart reçoit 12 louis d'or, une coquette somme pour l'époque.

13. Il s'agit d'une crypte archéologique qui conserve, encore aujourd'hui, les vestiges de mausolées, d'amphores funéraires, de sarcophages de toutes tailles et des sépultures; le tout s'étendant $\mathrm{du} \mathrm{V}^{\mathrm{e}}$ au XVIII ${ }^{\mathrm{e}}$ siècles.

14. Pour aller plus loin sur ce sujet, on peut lire : Sabine Barles, «Les villes transformées par la santé, XVIII"-XX siècles ", Les Tribunes de la santé, vol. 3, nº 4, 2011, p. 31-37.

15. Daniel Rabreau, Les dessins d'architecture au XVIII siècle, Paris, Bibliothèque de l'Image, 2003, p. 16.

16. Christian Taillard, Victor Louis (1731-1800): Le triomphe du goût français à l'époque néo-classique, Paris, Presses de l'Université Paris-Sorbonne, 2009, p. 178.

17. Le dictionnaire de l'Académie française nous livre une définition intéressante de cette formation dite «sur le tas »: «il s'agit d'une formation qui se fait directement sur le lieu de construction, qui s'acquiert, se fait par l'expérience directe du métier. Cet emploi dérive du sens familier de tas en maçonnerie : endroit où l'on taille les pierres à bâtir, puis où l'on construit le 
mur ». Lien URL : https://www.academie-francaise.fr/apprendre-sur-le-tard-et-apprendre-surle-tas, consulté le 20 mars 2021.

18. Pour aller plus loin sur ces sujets, on peut lire, Christian Taillard, Victor Louis (1731-1800) : Le triomphe du goût français à l'époque néo-classique, Paris, Presses de l'Université Paris-Sorbonne, 2009, p. 167-216.

19. A.M.B, série DD, liasse $36 \mathrm{~g}$, honoraires du Grand-Théâtre, année 1775 , mandement du 10 juin 1775.

20. Dom Devienne, Histoire de la Ville de Bordeaux, Première Partie Contenant Les Evénements Civils Et La Vie De Plusieurs Hommes Célèbres, Les Mœurs Et Coutumes Des Bordelais, Bordeaux, Simon de La Court, Première Édition, 1771, 537 pages. De son vrai nom Charles Jean-Baptiste d'Agneaux de Vienne (1728-1792). ABM, série AA, liasse 29 c, l'Histoire de Bordeaux de Dom Devienne. Ce vaste projet de publication est inauguré dans le courant de l'année 1752. La congrégation du moine perçoit alors plus de six mille livres, entre 1754 et 1758, pour l'aboutissement de ce projet. Mais, le travail est interrompu pendant un peu plus de 11 années et il faut attendre 1770 pour que la Ville décide enfin de lancer l'impression du premier volume, qui couvre l'histoire de Bordeaux, de l'Antiquité à la fin du XVIII siècle. Bien qu'aujourd'hui scientifiquement discutable et incomplète, cette tentative historiographique n'en demeure pas moins, pour l'époque, tout à fait méritoire.

21. ABM, série AA, liasse 29 c, l'Histoire de Bordeaux de Dom Devienne, lettre de Dom Devienne aux Jurats de la ville, 26 juin 1771.

22. ABM, série AA, liasse 29 c, l'Histoire de Bordeaux de Dom Devienne, lettre Dom Devienne aux Jurats de la ville, 5 janvier 1772. Il est aisé d'imaginer que selon son habitude, et faute de temps aussi, peut-être, Bonfin - soucieux de faire collaborer Dufart - soit intervenu à la fois auprès de la Ville mais aussi auprès de Dom Devienne, pour intégrer son dessinateur dans le projet.

23. ABM, série AA, liasse $29 \mathrm{c}$, l'Histoire de Bordeaux de Dom Devienne. L'accord entre Dom Devienne et Lagardette est passé le 12 juin 1771.

24. En quelques années, ces terrains sont devenus très attractifs car, grâce à l'économie florissante du port, la ville ne cesse de grandir, notamment vers ses faubourgs. Dans cette évolution, le quartier du Palais Gallien devient de plus en plus convoité et cette imposante ruine commence à gêner l'évolution urbanistique d'un nouveau quartier en devenir. Si, finalement, le projet de Lhôte et Bonfin est délaissé, les deux hommes n'abandonneront jamais véritablement leurs intentions. Ainsi, lorsque l'antique ruine devient bien national sous la Révolution et que les autorités locales envisagent de la transformer en cimetière, ils s'y opposent fortement, considérant que ce «[...] champ mortuaire [...] serait trop petit et trop voisin des beaux quartiers [...]». En réalité, depuis février 1793, les deux associés ont obtenu l'autorisation de diviser le terrain en quatre îlots séparés par deux rues, destinés à la construction de biens privés ; un nouveau projet qui finalement ne se réalisera pas, faute de moyens. Sur cette affaire, on peut lire: Isabelle Roux, "L'urbanisme", Le Port des Lumières, architecture et art urbain, Bordeaux, 1780-1815, C.E.R.C.A.M, Bordeaux, 1989, p. 17-26.

25. Laurence Chevallier, Jean-Baptiste Dufart (1750-1820) et le milieu artistique bordelais, thèse de doctorat en histoire de l'art moderne, Bordeaux, 2010, tapuscrit.

26. Laurence Chevallier, Jean-Baptiste Dufart (1750-1820) et le milieu artistique bordelais, thèse de doctorat en histoire de l'art, Université Bordeaux-Montaigne, soutenue en novembre 2010, tapuscrit original, p. 365 et 369.

27. Pour aller plus loin sur cette dialectique dessin/chantier, on peut lire l'article de Cyrille Simonnet, "Dessin-chantier. Réflexions sur la genèse de l'œuvre architecturale", Genesis (Manuscrits-Recherche-Invention), $\mathrm{n}^{\circ}$ 14, 2000, p. 111-128.

28. Au sujet du Théâtre Français, on peut lire : Robert Coustet, «Le Théâtre Français », actes du colloque Victor Louis et le théâtre, scénographie, mise en scène et architecture théâtrale aux XVIII et XIX ${ }^{e}$ siècles, Paris, Centre Régional de Publication de Bordeaux, 1982, pp. 199-207 et Laurence 
Chevallier, Jean-Baptiste Dufart (1750-1820) et le milieu artistique bordelais, thèse de doctorat en histoire de l'art moderne, Bordeaux, 2010, tapuscrit, p. 173-191.

29. ABM, série $B B$, liasse 133, délibérations de la Jurade, de mai 1770 au 9 juillet 1773, incendié le 13 juin 1862, reconstitué en 1888, lettre des Jurats du $1^{\mathrm{er}}$ septembre 1770.

30. ABM, Ms 556, fonds Braquehaye, Académie de peinture, 1780-1800, 11 août 1787.

31. Ibid.

32. Pour aller plus loin sur ce sujet, on peut lire : Laurence Chevallier, «Jean-Baptiste Dufart, architecte bordelais : réseau et clientèle ", actes du colloque international Chalgrin et son temps. Architectes et architectures de l'Ancien Régime à la Révolution, Annales du Centre Ledoux, t. IX, William Blake and Co/Art\&Art, 2016, p. 107-114.

\section{RÉSUMÉS}

Cet article s'intéresse à la formation des architectes en agence et, plus spécifiquement, à la formation de Jean-Baptiste Dufart, à Bordeaux, entre 1768 et 1780 . Bien qu'incomplets et lacunaires, les documents archivistiques mobilisés pourraient-ils permettre de mieux comprendre comment cette formation était possible alors que l'architecte était l'employeur et, l'aspirant architecte, un employé salarié ? Pourraient-ils également permettre de mieux saisir les échanges et les relations engagés entre le chef du bureau et son subalterne ? Pourrait-on alors identifier les formes que pouvaient prendre les «leçons»? La place que tenait le dessin dans ce type de formation? Les opportunités qu'offrait cet apprentissage? Ce sont ces ébauches de questions, encore bien trop larges, que cet article se propose d'appréhender.

This article focuses on the training of architects in agencies and, more specifically, on the training of Jean-Baptiste Dufart in Bordeaux, between 1768 and 1780. Although incomplete and deficient, could the archival documents mobilized help to better understand how this training was possible when the architect was the employer and, the aspiring architect, an employee? Could they also help to better understand the exchanges and relations between the head of the office and his subordinate? Could we then identify the forms that the "lessons » could take? The place that the drawing held in this type of training? The opportunities offered by this learning? It is these sketches of questions, still far too broad, that this article.

Dieser Beitrag beschäftigt sich mit der Architektenausbildung innerhalb von Architekturbüros am Beispiel der Ausbildung von Jean-Baptiste Dufart in Bordeaux zwischen 1768 und 1780. Obwohl nur eine lückenhafte Überlieferung besteht, mögen die vorhandenen Archivalien die Frage klären, wie sich eine solche Ausbildung gestaltete, wenn der Architekt gleichzeitig Arbeitgeber war und der Lehrling sein Angestellter. Auch geht es darum, die Beziehungen und den Austausch zwischen dem Büroleiter und seinem Mitarbeiter zu beleuchten. Zudem soll auch betrachtet werden, inwiefern man hier von einer architektonische "Lehre" sprechen kann, welche Rolle die Zeichnung dabei einnahm und welche beruflichen Aussichten eine solche Ausbildung bot. Diese Fragen können in diesem Beitrag nur angerissen werden, mögen aber weitere Forschungsperspektiven eröffnen. 
INDEX

Mots-clés : formation, architecture, XVIIIe siècle, Bordeaux, Jean-Baptiste Dufart

\section{AUTEUR}

\section{LAURENCE CHEVALLIER}

Laurence Chevallier est docteure en histoire de l'art et maîtresse de conférences en histoire et cultures architecturales à l'École nationale supérieure d'architecture et de paysage de Bordeaux. Membre du laboratoire CNRS PASSAGES (UMR 5319 - ENSAP-Bordeaux) et du centre FrançoisGeorges Pariset (EA538 - Université Bordeaux Montaigne), elle développe des thèmes de recherche centrés sur l'histoire de l'architecture en Nouvelle Aquitaine (XVIII ${ }^{\mathrm{e}}-\mathrm{XX}^{\mathrm{e}}$ siècles), les itinéraires d'architectes (XVIII ${ }^{\mathrm{e}}-\mathrm{XX}^{\mathrm{e}}$ siècles), la culture architecturale, les bibliothèques d'architectes et les réseaux et clientèles. Elle travaille aussi sur l'histoire de l'enseignement de l'architecture avec notamment la participation à deux programmes de recherches : le programme national HEnsA20 (2016-2021) et le programme ANR EnsArchi (2019-2022) et sur l'histoire du patrimoine local, ordinaire, rural, viti-vinicole et de montagne en étant, notamment, associée au programme FEDER TCV-PYR (2017-2021). 